\title{
Efeito dos anti-inflamatorios nas convulsões induzidas por pentilenotetrazol no modelo de Kindling: uma revisão
}

\author{
Effect of anti-inflammators on seizures induced by pentylenetetrazole in the Kindling model: a \\ review
}

Efecto de los antiinflamadores sobre las convulsiones inducidas por pentilenetetrazol en el modelo Kindling: una revisión

Recebido: 08/10/2021 | Revisado: 18/10/2021 | Aceito: 19/10/2021 | Publicado: 21/10/2021

\author{
Nicole Magarefo Barco \\ ORCID: https://orcid.org/0000-0001-6554-6299 \\ Centro Universitário da Fundação Assis Gurgacz, Brasil \\ E-mail: nicolebarco@icloud.com \\ Vagner Fagnani Linartevichi \\ ORCID: https://orcid.org/0000-0002-2624-7744 \\ Centro Universitário da Fundação Assis Gurgacz, Brasil \\ E-mail: linartevichi@fag.edu.br
}

\begin{abstract}
Resumo
Introdução: Estudos com anti-inflamatórios esteroidais e não esteroidais em modelo animal tem sido realizados a fim de verificar novas abordagens terapêuticas para o tratamento da epilepsia para melhorar a qualidade de vida dos pacientes portadores desta doença que acomete cerca de 65 milhões de pessoas no mundo. Objetivo: analisar a relevância no tratamento das crises convulsivas destes medicamentos. Metodologia: o presente estudo trata-se de uma revisão de literatura a fim de avaliar os efeitos dos anti-inflamatórios nas crises convulsivas. Resultado: os resultados obtidos demonstram que os animais tratados com os anti-inflamatórios esteroidais e não esteroidais estudados tiveram uma redução de intensidade nas crises convulsivas bem mais baixas sem evolução para a escala 4 de Racine. Conclusão: Conclui-se que os medicamentos anti-inflamatórios esteroidais e não esteroidais estudados tem o efeito protetivo e redutivo nas crises inflamatórias induzidas por pentilenotetrazol diminuindo as citocinas inflamatórias no sistema nervoso central.
\end{abstract}

Palavras-chave: Epilepsia; Anti-inflamatórios; Modelos animais.

\begin{abstract}
Introduction: Studies with steroidal and non-steroidal anti-inflammatory drugs in animal models have been carried out in order to verify new therapeutic approaches for the treatment of epilepsy to improve the quality of life of patients with this disease that affects about 65 million people worldwide. Objective: to analyze the relevance of these medications in the treatment of seizures. Methodology: this study is a literature review in order to assess the effects of anti-inflammatory drugs in seizures. Results: the results obtained demonstrate that the animals treated with the steroidal and non-steroidal anti-inflammatory drugs studied had a much lower intensity reduction in convulsive crises without progressing to the Racine 4 scale. Conclusion: It is concluded that the studied steroidal and non-steroidal antiinflammatory drugs have a protective and reductive effect on inflammatory crises induced by pentilenotetrazol, decreasing the inflammatory cytokines in the central nervous system.
\end{abstract}

Keywords: Epilepsy; Anti-inflammatory Agents; Animal models.

\section{Resumen}

Introducción: Se han realizado estudios con antiinflamatorios esteroideos y no esteroideos en modelos animales con el fin de verificar nuevos enfoques terapéuticos para el tratamiento de la epilepsia para mejorar la calidad de vida de los pacientes con esta enfermedad que afecta a cerca de 65 millones de personas en todo el mundo. Objetivo: analizar la relevancia de estos medicamentos en el tratamiento de las convulsiones. Metodología: este estudio es una revisión de la literatura con el fin de evaluar los efectos de los fármacos antiinflamatorios en las convulsiones. Resultados: los resultados obtenidos demuestran que los animales tratados con los antiinflamatorios esteroideos y no esteroideos estudiados presentaron una reducción de intensidad mucho menor en las crisis convulsivas sin progresar a la escala Racine 4. Conclusión: Se concluye que los antiinflamatorios esteroideos y no esteroideos estudiados tienen un efecto 
protector y reductor sobre las crisis inflamatorias inducidas por pentilotetrazol, disminuyendo las citocinas inflamatorias en el sistema nervioso central.

Palabras clave: Epilepsia; Antiinflamatorio; Modelos animales.

\section{Introdução}

A epilepsia é uma doença neurológica comum que se caracteriza por ocorrências de crises epilépticas que podem se repetir em intervalos de tempo variáveis. As crises de epilépticas são um tipo de manifestação clínica de uma descarga elétrica não comum de neurônios. A doença em fase crônica pode ter várias causas e que variam de acordo com o tipo de epilepsia que o paciente apresenta, bem como sua idade e sexo. Por exemplo em crianças a epilepsia pode aparecer por conta da anóxia neonatal e em idosos por doenças vasculares cerebrais como o AVC ou tumores cerebrais que são os mais frequentes (Marchetti et al., 2005).

Existem tipos de crises convulsivas descritas na literatura que se diferenciam, são elas, as Crises Parciais e Crises Generalizadas. Cada uma delas são adicionalmente divididas em subtipos. No caso de crises epilépticas parciais a subdivisão baseia-se na consciência que é perdida nas mais complexas e preservada nas mais simples e que mais tarde pode ser que evolua para uma crise generalizada. As crises epilépticas generalizadas estão subdivididas em tônico-clônica e crise de ausência (Minella \& Linartevichi, 2021). As crises parciais simples se caracterizam como aqueles que a descarga começa localmente e sua frequência tende a permanecer localizada, os seus sintomas dependem da região cerebral atingida pela descarga e envolvem contrações neuromusculares que são involuntárias, os efeitos da descarga podem ser observados pela experiência sensorial ou pelo humor e comportamento, sendo assim denominada de epilepsia psicomotora, já as crises parciais complexas há perda de consciência e o paciente fica mais confuso e 8 letargo e apresenta comportamentos espontâneos como caminhar, sentar, falar, levantar e olhar fixamente para algo (Pedley et al., 2000).

As crises generalizadas caracterizam-se por envolver todo o cérebro e abrange também o sistema reticular que efetua grande parte da atividade elétrica anormal. Neste tipo de crise epiléptica há perda imediata da consciência que é característica direta da crise generalizada, que se divide em tônico-clônica e crise ausência. Crise tônico-clônica compreende-se em uma contração forte e inicial de toda sua musculatura e causa um tipo de espasmo, a respiração para e a micção, defecação e salivação ocorrem com constância, ocorre a perda da consciência abruptamente e geralmente ocorre em duas fases separadamente a fase tônica quando há um aumento bilateral do tronco e membros e clônica que acompanha uma certa entonação alta, o chamado grito epiléptico que é seguido por abalos musculares sincrônicos, essa fase permanece por aproximadamente 1 minuto e o paciente continua inconsciente por mais 2-4 minutos, a recuperação é gradual e o paciente se sente doente e cansado, pode ocorrer algum tipo de lesão durante a crise convulsiva. Os ataques de ausência são mais comuns em crianças e são mais frequentes do que as crises tônico-clônicas, são mais fáceis de identificação visto que um de seus sintomas é a parada abrupta da atividade motora e cognitiva, fixação dos olhos em algum lugar e inconsciência das pessoas a sua volta, porem se recupera rapidamente e não possui efeitos consequentemente. O eletroencefalograma de um paciente com uma crise de ausência mostra uma descarga do tipo rítmica (Neto et al., 2019; Rang \& Dale., 2016).

Na área terapêutica da epilepsia, há uma grande necessidade de se desenvolver novos tratamentos antiepileptogênicos que ofereçam uma maneira de prevenir o aparecimento ou a progressão da doença. Esses tratamentos ainda são insuficientes e seu desenvolvimento requer um conhecimento profundo dos mecanismos subjacentes à patogênese da doença, a fim de direcioná-los ao uso de medicamentos apropriados com intervenções oportunas (Santos et al., 2014).

Descobertas emergentes destacam que moléculas inflamatórias específicas são alvos potenciais para intervenção medicamentosa para prevenir ou interromper a epileptogênese. Essas drogas, por interferirem nos mecanismos implicados no desenvolvimento da doença, podem representar tratamentos modificadores da doença (Garcia et al., 2020). A tradução clínica da intervenção anti-inflamatória pode tirar proveito de drogas já utilizadas na prática clínica para distúrbios periféricos ou 
outros do SNC com um componente neuroinflamatório patogênico. Atualmente estudos estão sendo desenvolvidos usando anti-inflamatórios esteroidais e não esteroides usando o modelo animal Kindling por pentilenotetrazol (PTZ). Citocinas de inflamação como a IL-1 $\beta$, TNF- $\alpha$ e IL-6 tem se mostrado aumentada nos modelos de experimento de convulsões das áreas cerebrais de propagação convulsiva, em destaque para a glia e em menor grau por neurônios (Vezzani et al., 2008).

O Sistema Nervoso Central (SNC) é imuno privilegiado por ser altamente especializado em se proteger de reações imunomediadas devido ao seu trafego reduzido de monócitos e linfócitos entre o SNC e o sangue. A barreira hematoencefálica (BHE) é uma estrutura tecidual que consiste em uma camada de células do endotélio que envolvem o cérebro, sua função permite a troca de água, íons, proteínas e ajuda na endocitose controlando o balanço hídrico do cérebro (Abbot et al., 1996).

Estudos experimentais em modelos de convulsões e epilepsia demonstraram que a inflamação do cérebro não é uma simples marca registrada da patologia do tecido, mas desempenha um papel ativo em sustentar ou precipitar convulsões e contribui para a perda de células (Rocha Júnior et al., 2021). Os mecanismos moleculares pelos quais a inflamação pode aumentar a excitabilidade do tecido são agora explorados, abrindo novas perspectivas atraentes para o tratamento ou prevenção de convulsões. Mediadores inflamatórios foram descritos recentemente em tecido ressecado cirurgicamente de pacientes farmacorresistentes com convulsões de várias etiologias. Essa observação amplia a visão original de que a inflamação é uma característica patológica de distúrbios epilépticos específicos, como a encefalite, para a possibilidade de que a inflamação do cérebro seja de fato ou um substrato patológico comum que contribui para a epileptogenicidade do tecido (Vezzani et al., 2012).

A inflamação desempenha um papel importante no processo de epileptogênese, no qual ocorre redução do limiar de convulsão. Vários mediadores inflamatórios com propriedades epileptogênicas incluindo a interleucina $1 \beta$ (IL-1ß) são ativadas fazendo com que haja aumento da permeabilidade da barreira hematoencefálica (BHE), estas ações desempenham papeis importantes no desenvolvimento da epilepsia, ou seja, os processos inflamatórios podem ser causa ou a consequência das crises epilépticas (Guzzo., 2019).

Existem algumas citocinas responsáveis por transportar a resposta inflamatória nos locais de lesão, por exemplo as interleucinas, alguns fatores de TNF-alfa dos quais são amplamente utilizados em pesquisa para classificar os estados inflamatórios. A interleucina 1 e 6 e TNF-alfa são as mais importantes neste segmento por terem ação pró-inflamatório (McLaren et al., 2011).

A interleucina 1 (IL-1) é uma importante citocina mediadora de respostas inflamatórias produzida por células epiteliais, endoteliais e neutrófilos. Ela está presente no organismo em duas formas diferentes como a IL-1alfa e IL-1beta, exercendo papeis parecidos. De modo geral a IL-1 está associada a produção de linfócitos e a IL-1beta estimula a atividade de prostaglandina podendo levar ao estado de febre. Sugere-se ainda que a IL-1beta induz alguns episódios de convulsões através da regulação dos receptores de NDMA em células pós-sinápticas através de uma ativação da subunidade GluN2B do receptor NDMA (Rana et al., 2018).

A interleucina 6 (IL-6) é considerada a mais importante citocina em respostas inflamatórias pois apresenta efeitos sistêmicos e locais além de estimular a produção de neutrófilos. É sintetizada especialmente por células endoteliais vascularizadas e fagócitos mononucleares. Estudos mostraram que os níveis de IL-6 têm predomínio de aumento no soro de pacientes com epilepsia comparado a outros sem a doença. Também foi encontrado o aumento da citocina no LCR (liquido cefalorraquidiano) de cães que apresentavam crises epilépticas comparando com cães saudáveis, além disso também se observou o aumento de TNF-alfa (Jiang., 2018; Allan., 2001).

O TNF-alfa é um mediador de respostar inflamatórias a bactérias e outros organismos infecciosos. É uma proteína sintetizada por monócitos, macrófagos e células dendríticas. Esta citocina tem como função regular os receptores de glutamato alfa-amino-3-hidroxi-metil-5-4-isoxazolpropiónico (AMPA) fazendo com que haja aumento glutamatérgico. Esses receptores 
quando aumentados aumentam a absorção de cálcio fazendo com que ocorra uma neurotoxicidade. Essa citocina multiplica o tanto de receptores de glutamato, mas também induz a endocitose do receptor do GABA que reduz o impulso inibitório causando baixa excitabilidade celular, assim compreende-se que a TNF-alfa possui propriedades neuromodulatórias pelo fato de ocorrer essas excitabilidades celular (Idriss, 2000; Rana et al., 2018).

Os estudos do processo inflamatório juntamente com os mediadores inflamatórios na patogênese da epilepsia tem sido alvo de vários estudos clínicos envolvendo modelo animal. O modelo animal mais utilizado são os ratos (Fagundes et al., 2004). Para a identificação de possíveis agentes terapêuticos para o tratamento da epilepsia, usa-se o modelo animal de crise epiléptica crônica. É usado o eletrochoque e administrado substancias químicas moduladoras da convulsão (pró-convulsivas). O modelo mais utilizado para observar estes parâmetros é o modelo de Kindling (Löscher, 2017).

O modelo Kindling é considerado um modelo crônico de convulsões (de Lima Rosa et al, 2021). É utilizado o PTZ, uma substancia que induz as crises convulsivas amplamente utilizado em modelos animais para investigações envolvendo crises convulsivas (Mussulini, 2013). O PTZ atua como um antagonista dos receptores GABAérgicos, bloqueando o influxo de íons de cloreto através desses canais e aumentando a excitabilidade neuronal e convulsões (de Lima Rosa et al., 2021).

A gravidade das tais crises epilépticas nos modelos experimentais da epilepsia é frequentemente avaliada por meio da Escala de Racine, que apesar dos seus métodos de indução de convulsões com o uso de PTZ difere do artigo original de Racine em 1972. No entanto muitos outros métodos de indução da epilepsia são implementados nesta pesquisa os principais são a aplicação de produtos químicos como o próprio PTZ e a Estricnina ou estímulo elétrico de modelos de crises crônicas como ácido caínico e a Pilocarpina (Van Erun et al., 2019). Esta escala é graduada de 1 a 5, sendo respectivamente observados os seguintes comportamentos: normal, hiperatividade, clônica dos membros anteriores, clônica dos membros posteriores, ataque motor completo. Assim, neste cinco estágios, os dois primeiros são referentes as crises iniciais e focais, o estágio três e quatro são para convulsões parciais e de generalização secundaria e o último estágio é definido para crises de movimentos clônicos acompanhados por perda de tônus postural (Racine, 1972).

Neste contexto, o presente trabalho teve por objetivo fazer uma revisão descritiva-exploratória da literatura sobre os estudos envolvendo os medicamentos anti-inflamatórios no tratamento da epilepsia induzida pelo modelo Kindling.

\section{Metodologia}

Para a realização deste estudo, escolheu-se a metodologia de revisão descritiva-exploratória de literatura (Estrela, 2018), cujo intuito foi apresentar considerações já elaboradas, a fim de que os resultados sejam atualizados, pautando-se em estudos mais antigos, para que novas considerações sejam viabilizadas.

Deste modo, foram realizadas 3 etapas para a elaboração do material, baseada na pergunta norteadora: Quais são os efeitos dos anti-inflamatórios nas crises induzidas em roedores por PTZ avaliados no método Kindling? Primeiramente, realizou-se uma busca geral nas plataformas de pesquisa acadêmica Scielo e Pubmed, com base nos seguintes Descritores em Ciências da Saúde (DeCS): método Kindling, pentilenotetrazol, epilepsia e convulsões, todos nas opões AND e OR.

Após, aplicaram-se critérios mais específicos para a seleção da amostragem, baseados no período correspondente a 2008-2021, cuja escrita encontrava-se em língua portuguesa ou inglesa. Foram incluídos materiais que contemplavam em sua metodologia revisões sistemáticas, estudos de caso, estudos descritivos transversais e experimentais (dados organizados segundo Minella \& Linartevichi, 2021).

Inicialmente, encontrou-se um total de 210 artigos que continham todos os descritores supracitados. Em uma análise precisa a partir da leitura dos resumos foram descartados os trabalhos que não contemplavam o objetivo, que não expunham resultados diante da utilização dos anti-inflamatórios no método estudado, restando 18 artigos. Seguindo uma análise ainda mais minuciosa, consistindo na leitura integral e fichamento do material, foram selecionados 5 artigos que estavam dentro dos 
critérios da revisão descritivo-exploratória e que atendiam aos objetivos e à justificativa proposta pelo estudo.

\section{Resultados e Discussão}

Após a busca na literatura, apenas 5 estudos publicados entre 2008 a 2021 foram encontrados e selecionados por se encaixarem nos parâmetros desejados, destacando o teste com anti-inflamatórios sobre as convulsões. Na Tabela 1 estão sumarizados os artigos que compõe o presente estudo.

Tabela 1: Artigos de testes clínicos envolvendo o uso de anti-inflamatórios no modelo de Kindling.

\begin{tabular}{|c|c|c|c|c|}
\hline Estudo & Medicamento & Modelo & Animais & Achados principais \\
\hline $\begin{array}{l}\text { Rosa et al, } \\
2020\end{array}$ & Prednisolona & $\begin{array}{l}\text { Kindling (os ratos receberam } \\
25 \mathrm{mg} / \mathrm{kg} \text { de PTZ } 30 \text { minutos } \\
\text { após os outros tratamentos e } \\
\text { foram observados por } 20 \\
\text { minutos para avaliar a } \\
\text { gravidade das convulsões } \\
\text { utilizando a escala de Racine) }\end{array}$ & $\begin{array}{l}\text { Ratos wistar machos com } \\
2 \text { meses de idade pesando } \\
\text { aproximadamente } 300 \mathrm{~g} .\end{array}$ & $\begin{array}{l}\text { As doses de Prednisolona de } \\
1 \mathrm{mg} / \mathrm{kg} \text { e } 5 \mathrm{mg} / \mathrm{kg} \text { teve um efeito } \\
\text { protetor nas crises convulsivas. O } \\
\text { uso de Diazepam levou a } \\
\text { intensidades mais baixas das crises } \\
\text { quando comparados com o grupo de } \\
\text { solução salina. }\end{array}$ \\
\hline $\begin{array}{l}\text { Guzzo et al, } \\
2018\end{array}$ & Dexametasona & $\begin{array}{l}\text { Kindling (os ratos receberam } \\
\text { doses de } 20 \mathrm{mg} / \mathrm{kg} \text { de PTZ após } \\
\text { receberem o tratamento) foram } \\
\text { observados por } 30 \text { min e } \\
\text { avaliou-se a gravidade das } \\
\text { convulsões. }\end{array}$ & $\begin{array}{l}\text { Ratos Wistar, machos com } \\
2 \text { meses de idade pesando } \\
250-300 \mathrm{~g} .\end{array}$ & $\begin{array}{l}\text { As intensidades das convulsões } \\
\text { foram reduzidas nos animais que } \\
\text { utilizaram a Dexametasona }(1,2,3 \text { e } \\
4 \mathrm{mg} \text { ) e Diazepam } 2 \mathrm{mg} \text {. Nenhum } \\
\text { teve evolução para a escala } 4 \text { de } \\
\text { Racine. }\end{array}$ \\
\hline $\begin{array}{l}\text { Vieira et al, } \\
2016\end{array}$ & $\begin{array}{l}\text { Diclofenaco } \\
\text { Sódico }\end{array}$ & $\begin{array}{l}\text { Kindling (os ratos receberam } \\
20 \mathrm{mg} / \mathrm{kg} \text { de PTZ após } 30 \mathrm{~min} \\
\text { depois de receber o tratamento, } \\
\text { e avaliou-se a gravidade das } \\
\text { convulsões. }\end{array}$ & $\begin{array}{l}\text { Ratos Wistar } 3 \text { meses de } \\
\text { idade pesando } 300 \mathrm{~g} \text {. }\end{array}$ & $\begin{array}{l}\text { As intensidades das convulsões } \\
\text { foram reduzidas no grupo de ratos } \\
\text { tratados com Diclofenaco } \\
\text { Sódico. }\end{array}$ \\
\hline $\begin{array}{l}\text { Oliveira et al, } \\
2008\end{array}$ & Celecoxibe & $\begin{array}{l}\text { Kindling (os ratos receberam } \\
\text { doses de } 60 \mathrm{mg} / \mathrm{kg} \text { de PTZ após } \\
\text { o tratamento e foram } \\
\text { observados a intensidade das } \\
\text { convulsões. }\end{array}$ & $\begin{array}{l}\text { Ratos wistar, machos e } \\
\text { adultos. }\end{array}$ & $\begin{array}{l}\text { O medicamento teve ação protetora } \\
\text { nas convulsões induzidas. }\end{array}$ \\
\hline $\begin{array}{l}\text { Akula et al, } \\
2008\end{array}$ & Rofecoxibe & $\begin{array}{l}\text { Kindling (os ratos receberam } \\
\text { doses de PTZ preparado a } 0,5 \% \\
\text { em solução salina. }\end{array}$ & $\begin{array}{l}\text { Camundongos albinos, } \\
\text { machos pesando entre } 22 \mathrm{e} \\
30 \mathrm{~g}\end{array}$ & $\begin{array}{l}\text { O pré tratamento de } 4 \mathrm{mg} / \mathrm{kg} \\
\text { diminuiu significativamente a } \\
\text { intensidade das convulsões } \\
\text { induzidas, já na dosagem de } \\
2 \mathrm{mg} / \mathrm{kg} \text { houve aumento do limiar } \\
\text { convulsivo. }\end{array}$ \\
\hline
\end{tabular}

Fonte: Autores.

No estudo de Vieira e colaboradores (2016), com Diclofenaco sódico, os ratos foram divididos em três grupos, o primeiro e o segundo receberam doses de $5 \mathrm{mg} / \mathrm{kg}$ e $10 \mathrm{mg} / \mathrm{kg}$ de Diclofenaco sódico e o terceiro grupo recebeu Diazepam $2 \mathrm{mg} / \mathrm{kg}$ via intraperitoneal. Os três grupos de ratos receberam 20mg/kg de PTZ após 30 minutos depois de receberem as doses terapêuticas de Diclofenaco sódico e Diazepam e foi avaliado a intensidade das crises convulsivas. Segundo Vieira et al, (2016) a intensidade das crises convulsivas observadas foi reduzida nos grupos de ratos tratados com ambas as doses de 5 e $10 \mathrm{mg} / \mathrm{kg}$ de Diclofenaco sódico e $2 \mathrm{mg}$ de Diazepam. Nenhum animal evoluiu para o nível 4 da escala de Racine. Observou-se ainda que a dose mais baixa de Diclofenaco sódico também foi capaz de reduzir os níveis de IL-6, o aumento desta citocina no LCR principalmente está relacionado diretamente com as convulsões recorrentes de pacientes, e a elevação recorrente desta citocina pode danificar estruturas cerebrais e ocorrer comprometimento neurológico (Campbell et al., 1993).

Guzzo e colaboradores (2018), estudaram a Dexametasona, os ratos foram divididos em seis grupos onde submeteram doses de $1 \mathrm{mg} / \mathrm{kg}, 2 \mathrm{mg} / \mathrm{kg}, 3 \mathrm{mg} / \mathrm{kg}$ e $4 \mathrm{mg} / \mathrm{kg}$ de Dexametasona, o quinto grupo recebeu $2 \mathrm{mg} / \mathrm{kg}$ de Diazepam e o último grupo 
recebeu salina, todos via intraperitoneal. Os grupos receberam doses de $20 \mathrm{mg} / \mathrm{kg}$ de PTZ após receberem o tratamento com os medicamentos e foi observado a intensidade das crises convulsivas. Segundo Guzzo et al, (2018) a intensidade das crises convulsivas nos animais que utilizaram Dexametasona $1 \mathrm{mg} / \mathrm{kg}, 2 \mathrm{mg} / \mathrm{kg}, 3 \mathrm{mg} / \mathrm{kg}$ e $4 \mathrm{mg} / \mathrm{kg}$ e Diazepam $2 \mathrm{mg} / \mathrm{kg}$ foi reduzida tendo um efeito protetor diante da inflamação produzida pelas crises induzidas. Nenhum dos animais teve evolução para a escala 4 de Racine. Observou-se uma diminuição significante dos níveis de TNF- $\alpha$ no hipocampo dos animais tratados com a Dexametasona $4 \mathrm{mg} / \mathrm{kg}$ em comparação com os grupos de Dexametasona $1 \mathrm{mg} / \mathrm{kg}$ e $2 \mathrm{mg} / \mathrm{kg}$.

No estudo com a Prednisolona, os ratos foram divididos em quatro grupos onde o grupo um e dois foram submetidos a doses de $1 \mathrm{mg} / \mathrm{kg}$ e $5 \mathrm{mg} / \mathrm{kg}$ de Prednisolona, o terceiro doses de $2 \mathrm{mg} / \mathrm{kg}$ de Diazepam e o quarto doses de salina a $0,9 \%$ via intraperitoneal. Os grupos também receberam doses de $25 \mathrm{mg} / \mathrm{kg}$ de PTZ após terem recebido o tratamento com os medicamentos e foi observado a intensidade das crises convulsivas. Segundo de Lima Rosa et al, 2020 as doses de Prednisolona de $1 \mathrm{mg} / \mathrm{kg}$ e $5 \mathrm{mg} / \mathrm{kg}$ tiveram um efeito protetor nas crises, e o Diazepam levou a um efeito mais baixo nas crises de convulsão induzidas quando comparado com o grupo que recebeu a solução de salina a 0,9\%. A Prednisolona teve um efeito protetor contra os níveis das citocinas inflamatórias IL-1 $\beta$, IL-6 e TNF- $\alpha$ (de Lima Rosa et al., 2021).

No estudo de Oliveira 2008 com o Celecoxibe, os ratos foram divididos em quatro grupos onde foram submetidos a doses de $0,2 \mathrm{mg} / \mathrm{kg}, 2 \mathrm{mg} / \mathrm{kg}$ e $20 \mathrm{mg} / \mathrm{kg}$ de Celecoxibe, no quarto grupo foi administrado doses de PGE2 via intraperitoneal. Os grupos receberam doses de $60 \mathrm{mg} / \mathrm{kg}$ de PTZ após o tratamento com o medicamento e assim foram observadas as intensidades das crises convulsivas. Este quarto experimento com a PGE2 é feito com o objetivo de avaliar se a mesma causa atividade convulsiva ou se facilita a ação convulsiva de PTZ. O Celecoxibe teve ação anticonvulsivante pois foi revertido pelo método intracerebroventricular de administração de PGE2.

No estudo de Akula 2008 com o Rofecoxibe, os ratos foram divididos em dois grupos onde foram submetidos a doses de $2 \mathrm{mg} / \mathrm{kg}$ e $4 \mathrm{mg} / \mathrm{kg}$ de Rofecoxibe suspenso em $0,5 \%$ de carboximetilcelulose via intraperitoneal. Receberam doses de PTZ preparado em $0,5 \%$ de solução salina infundida por via intravenosa e assim foram observados a intensidade das convulsões induzidas. O Rofecoxibe teve ação anticonvulsivante a partir da dose de $4 \mathrm{mg} / \mathrm{kg}$ pois houve o aumento do limiar convulsivo, nas doses menores apresentou pouca eficiência perante o nível do limiar convulsivo.

\section{Considerações Finais}

Novas abordagens têm sido estudadas a fim de resolver os problemas e melhorar a qualidade de vida de pacientes epilépticos, tais abordagens surgem com os avanços dos estudos experimentais de pesquisas pré-clínicas e clínicas utilizando alguns medicamentos anti-inflamatórios selecionados, visto que estes estudos demonstraram que a epilepsia está influenciada na inflamação do processo epileptogênico.

Conforme os estudos experimentais descritos neste trabalho utilizando os modelos animais de Kindling a fim de se verificar-se o efeito dos anti-inflamatórios esteroidais e não esteroidais no processo de proteção nas crises induzidas por PTZ, conclui-se que houve um grande avanço no tratamento das crises convulsivas e um importante índice protetor da neuroinflamação diminuindo os níveis das citocinas pró-inflamatórias no sistema nervoso central.

A partir disso entende-se que novos estudos devem ser executados, sejam eles metanálises ou experimentais para que haja melhoria, no futuro, no tratamento dos portadores de epilepsia. O reposicionamento dos fármacos já existentes no mercado se mostra muito interessante, uma vez que, atuando em diferentes patologias, diminuem o número de medicamentos administrados, favorece a adesão ao tratamento e pode proporcionar uma melhor qualidade de vida aos portadores de epilepsia. 


\section{Referências}

Abbott, N. J., \& Romero, I. A. (1996). Transporting therapeutics across the blood-brain barrier. Molecular Medicine Today, 2(3), 106-113. https://doi.org/10.1016/1357-4310(96)88720-X

Akula, K. K., Dhir, A., \& Kulkarni, S. K. (2008). Rofecoxib, a selective cyclooxygenase-2 (COX-2) inhibitor increases pentylenetetrazol seizure threshold in mice: possible involvement of adenosinergic mechanism. Epilepsy Research, 78(1), 60-70. https://doi.org/10.1016/j.eplepsyres.2007.10.008

Allan, S. M., \& Rothwell, N. J. (2001). Cytokines and acute neurodegeneration. Nature Reviews Neuroscience, 2(10), 734-744. https://doi.org/10.1038/35094583

Campbell, I. L., Abraham, C. R., Masliah, E., Kemper, P., Inglis, J. D., Oldstone, M. B., \& Mucke, L. (1993). Neurologic disease induced in transgenic mice by cerebral overexpression of interleukin 6. Proceedings of the National Academy of Sciences of the United States of America, 90(21), 10061-10065. https://doi.org/10.1073/pnas.90.21.10061

de Lima Rosa, G., Muller Guzzo, E., Muliterno Domingues, A., Bremm Padilha, R., Dias de Oliveira Amaral, V., \& Simon Coitinho, A. (2021). Effects of prednisolone on behavioral and inflammatory profile in animal model of PTZ-induced seizure. Neuroscience Letters, 743, 135560. https://doi.org/10.1016/j.neulet.2020.135560

Estrela, C. (2018). Metodologia Científica: Ciência, Ensino, Pesquisa. Editora Artes Médicas.

Fagundes, J. D, Taha, O. M. (2004) Animal disease model: choice's criteria and current animals specimens. Acta Cirurgica Brasileira, 19 (1), 59-65. https://doi.org/10.1590/S0102-86502004000100010

Garcia, T. R., Cruz, M. C. A, Silva, G. de O. A., Cardoso, E. F., \& Arruda, J. T. (2020). Cannabidiol for the treatment of patients with West syndrome and epilepsy. Research, Society and Development, 9(9), e420997267. https://doi.org/10.33448/rsd-v9i9.7267

Guzzo, E., Lima, K. R., Vargas, C. R., \& Coitinho, A. S. (2018). Effect of dexamethasone on seizures and inflammatory profile induced by Kindling Seizure Model. Journal of Neuroimmunology, 325, 92-98. https://doi.org/10.1016/j.jneuroim.2018.10.005

Idriss, H. T., \& Naismith, J. H. (2000). TNF alpha and the TNF receptor superfamily: structure-function relationship(s). Microscopy Research and Technique, 50(3), 184-195. https://doi.org/10.1002/1097-0029

Jiang, N. M., Cowan, M., Moonah, S. N., \& Petri, W. A., Jr (2018). The Impact of Systemic Inflammation on Neurodevelopment. Trends in Molecular Medicine, 24(9), 794-804. https://doi.org/10.1016/j.molmed.2018.06.008

Kingsley, P. J., Rouzer, C. A., Morgan, A. J., Patel, S., \& Marnett, L. J. (2019). Aspects of Prostaglandin Glycerol Ester Biology. Advances in Experimental Medicine and Biology, 1161, 77-88. https://doi.org/10.1007/978-3-030-21735-8_8

Löscher W. (2017). Animal Models of Seizures and Epilepsy: Past, Present, and Future Role for the Discovery of Antiseizure Drugs. Neurochemical Research, 42(7), 1873-1888. https://doi.org/10.1007/s11064-017-2222-z

McLaren, J. E., Michael, D. R., Ashlin, T. G., \& Ramji, D. P. (2011). Cytokines, macrophage lipid metabolism and foam cells: implications for cardiovascular disease therapy. Progress in Lipid Research, 50(4), 331-347. https://doi.org/10.1016/j.plipres.2011.04.002

Minella, F. C. O., \& Linartevichi, V. F. (2021). Effects of cannabidiol on the signs and comorbidities of autistic spectrum disorder. Research, Society and Development, 10(10), e64101018607. https://doi.org/10.33448/rsd-v10i10.18607

Mussulini, B. H. M. (2013) Caracterização comportamental do modelo de convulsões induzidas por pentilenotetrazol em zebrafish adulto. UFRGS (Universidade Federal do Rio Grande do Sul)

Neto, H., Chagas, B., Soares, M., Lachinski, R., \& Linartevichi, V. (2019). Síndrome de Stevens-Johnson associada a Fenitoína em pós-operatório de hemorragia intraparenquimatosa cerebral: relato de caso. Fag Journal of Health, 1(4), 169-184. https://doi.org/10.35984/fjh.v1i4.110

Neto, J. G, Marchetti, R. L. (2005) Aspectos epidemiológicos e relevância dos transtornos mentais associados à epilepsia. Brazilian Journal of Psychiatry, 27 (4), 323-328. https://doi.org/10.1590/S1516-44462005000400013

Oliveira, M. S., Furian, A. F., Royes, L. F., Fighera, M. R., Fiorenza, N. G., Castelli, M., Machado, P., Bohrer, D., Veiga, M., Ferreira, J., Cavalheiro, E. A., \& Mello, C. F. (2008). Cyclooxygenase-2/PGE2 pathway facilitates pentylenetetrazol-induced seizures. Epilepsy Research, 79(1), 14-21. https://doi.org/10.1016/j.eplepsyres.2007.12.008

Pedley, A. Timothy, B. W. Carl, Morrell, J. Martha. (2000) Epilepsia - Merritt Tratado de Neurologia.

Racine R. J. (1972). Modification of seizure activity by electrical stimulation. II. Motor seizure. Electroencephalography and Clinical Neurophysiology, 32(3), 281-294. https://doi.org/10.1016/0013-4694(72)90177-0

Rana, A., \& Musto, A. E. (2018). The role of inflammation in the development of epilepsy. Journal of Neuroinflammation, 15(1), 144. https://doi.org/10.1186/s12974-018-1192-7

Rang, H. P. Dale, M. M. Ritter, J. M. Flower, R. J. Henderson, Goodman \& Gilman. (2018) As Bases Farmacológicas da Terapêutica. (13a ed.), McGraw-Hill, 2018 .

Rocha Junior, F. C. de M.., Alencar Pereira, G. J., Santos, H. R. de O.., Oliveira, R. M. R., Pedroso Rodríguez, M. de los Ángeles, \& López, S. G. (2021). Learning disorder and epilepsy. Research, Society and Development, 10(3), e1910313039. https://doi.org/10.33448/rsd-v10i3.13039

Santos, D. R. (2014) A epilepsia e seus principais tratamentos. Faema. 30p. Ariquemes - RO. 
Research, Society and Development, v. 10, n. 13, e538101321581, 2021

(CC BY 4.0) | ISSN 2525-3409 | DOI: http://dx.doi.org/10.33448/rsd-v10i13.21581

Van Erum, J., Van Dam, D., \& De Deyn, P. P. (2019). PTZ-induced seizures in mice require a revised Racine scale. Epilepsy \& Behavior: E\&B, 95, 51-55. https://doi.org/10.1016/j.yebeh.2019.02.029

Vezzani, A., Balosso, S., \& Ravizza, T. (2012). Inflammation and epilepsy. Handbook of Clinical Neurology, 107, 163-175. https://doi.org/10.1016/B978-0444-52898-8.00010-0

Vezzani, A., Balosso, S., \& Ravizza, T. (2008). The role of cytokines in the pathophysiology of epilepsy. Brain, Behavior, and Immunity, 22(6), 797-803. https://doi.org/10.1016/j.bbi.2008.03.009

Vieira, V., Glassmann, D., Marafon, P., Pereira, P., Gomez, R., \& Coitinho, A. S. (2016). Effect of diclofenac sodium on seizures and inflammatory profile induced by Kindling seizure model. Epilepsy Research, 127, 107-113. https://doi.org/10.1016/j.eplepsyres.2016.08.020 